\title{
Knee Pain in Adults \& Adolescents, Diagnosis and Treatment
}

\author{
Sherif Hosny, W. McClatchie, Nidhi Sofat and Caroline B. Hing
}

Additional information is available at the end of the chapter

http://dx.doi.org/10.5772/51077

\section{Introduction}

Knee pain is a common symptom affecting both children and adults [1]. Assessment of pain is important in order to determine the aetiology of the underlying condition which can be investigated with mechanistic studies further in order to assess the response to treatment interventions.

We performed a literature search to identify pertinent review articles investigating advances in knee pain diagnosis and management in humans using the $\mathrm{MeSH}$ terms and Boolean operators 'knee' AND 'pain'. We identified 11928 relevant articles and limited our search to review articles on humans in English. The structure of the chapter is divided into subheadings covering definition, incidence, aetiology, classification, diagnosis and management. Within each subheading, further sections address the use of novel imaging to quantify disease progression, operative and non-operative management. A separate section will outline the causes and treatment of knee pain in adolescents. A further section outlines future advances in diagnosis and management.

\section{Definition}

Knee pain is broadly divided into tibiofemoral and anterior knee pain (AKP). Knee pain can be referred from the hip or present as part of radiculopathy. AKP can refer to a number of symptoms. In the past the terms 'chondromalacia patellae' and 'patellofemoral pain' have been used loosely to describe such symptoms. This is to be avoided, as whilst both conditions are recognised, they may not be the cause of the pain. The term 'anterior knee pain' may be used when no specific diagnosis has been made [2].

Jackson et al (2001) divided the causes of AKP broadly into two groups [1]. The first group includes focal lesions which can be identified clinically or radiologically, and is described as 
distinct. This includes overuse syndromes, trauma related lesions, dysplasias, tumours, and iatrogenic lesions. The second group is described as obscure and includes types of knee pain that are dynamic, or are more difficult to define. The symptoms may vary widely between individuals with the same clinical findings. This includes patellar maltracking, chondromalacia, idiopathic pain, reflex sympathetic dystrophy and psychogenic pain.

Tibiofemoral knee pain can be described as acute or chronic. Acute symptoms are often mechanical in nature and due to a recent injury. Pain may arise from acute injury to the menisci, and / or to the ligamentous stabilisers of the knee. Resultant alteration to the biomechanics of the joint can lead to degenerative change and associated symptoms.

Chronic knee pain is commonly due to arthritic change. Osteoarthritis (OA) is the most common and is a degenerative condition caused by focal cartilage loss. It is often described according to conventional radiological findings as described by Kellgren and Lawrence [3]. These include osteophyte formation, narrowing of joint space, sclerosis of subchondral bone and cyst formation. Utilising these changes as a guide, five grades were used to describe OA: 1. None, 2. doubtful, 3. minimal, 4. moderate and 5. Severe [2]. Ahlbäck [4] described the radiographic changes in terms of joint space narrowing (Grades I-II) and bone attrition (Grades III - V). The American College of Rheumatology's classification of OA mentions knee pain occurring over most days of the previous month, crepitus on joint movement and morning stiffness. The World Health Organisation (WHO) Global Burden of Disease defined $\mathrm{OA}$ of the knee as a combination of these clinical criteria in the presence of defined radiological changes [5].

Rheumatoid arthritis (RA) is a destructive chronic inflammatory condition that affects the small joints of the hands and feet, along with larger joints. Symptoms initially include pain and swelling as the synovial lining of the joint is inflamed. Chronic progression of the disease leads to cartilage erosion and joint laxity resulting in deformity. Conversely, crystal arthropathy results from the deposition of monosodium urate (gout) or calcium pyrophosphate (pseudogout) within the knee joint. The precipitation of these crystal induces an inflammatory reaction within the joint.

\section{Incidence / prevalence}

There is some difficulty in establishing the true incidence of knee pain, as the actual definition of knee pain varies according to its cause. Most data available relates to arthritis, as it is a chronic disabling condition, with a significant impact on health. In a cross sectional study of 3341 residents aged over 65 in Oxfordshire, United Kingdom, the prevalence of knee pain was $32.6 \%$, using the SF-36 questionnaire [6].

There is a paucity of reports on the prevalence of knee pain from developed countries, Haq 2011 [7] reported global findings from the Community Orientated Program for Control Of Rheumatic Disorders (COPCORD). Wide variation in knee pain was seen both between rural and urban settings and also between countries. In all studies the prevalence of knee pain was greater in women than in men. Rural Iran had the highest recorded prevalence of $39.2 \%$ across the population. 
A cohort study by Ingham et 2011 [8] found the risk of knee pain for people over the age of forty in the United Kingdom was 32/1000 person-years (3.2\%) or a 12-year cumulative incidence of knee pain of $34.4 \%$ (32\% for men, 35\% for women). There was an increasing trend observed with increasing body mass index. The incidence in a Bangladeshi rural community recorded through COPCORD was only $6.5 \%$.

The incidence of reported knee pain in older patients is increasing, yet this does not always correlate with radiographic changes associated with arthritis. Nguyen et al 1999 [9] reported on three separate time periods measured by the Framingham Osteoarthritis Study in the US. This showed the prevalence of knee pain in men between 2002 and 2005 was 32.9\%, and $27.7 \%$ in women. An increasing trend in reported knee pain was observed over three decades in the over 70 age group. Surprisingly this trend did not correlate with an increasing body mass index (BMI), confounding the observation seen elsewhere that increased BMI correlates with an increased incidence of OA.

Symptomatic OA in younger patients was recorded by the 1987 Framingham study. Five percent of those over 26 years of age had pain along with radiological changes, with $6.7 \%$ of those over 45 years of age reporting the same symptoms. McAlindon et al 1992 [10] reported the prevalence of symptomatic isolated patellofemoral osteoarthritis of $11 \%$ in males over 55 years, and $24 \%$ in women over 55 years. In asymptomatic patients $3.8 \%$ of both men and women had isolated radiographic patellofemoral OA.

Jarvholm et al 2008 [11] reported that occupational factors are important risk factors for developing OA of the knee. In a population of male construction workers in Sweden the relative risk for surgically treated OA of the knee was 4.7. Those with more labour intensive roles had an increased risk. In a study of Finnish forestry workers the incidence of knee pain was $10 \%$ over a one year period [12]. Increased age, being overweight and knee straining activities all increased the likelihood of developing pain. The persistence of symptoms was associated with increased age and job dissatisfaction.

It is difficult to establish the exact incidence of knee pain specifically due to RA, although there appears to be a decreasing trend in knee arthroplasty for these patients. In a study of Californian adults over two decades Louie et al 2010 [13] noted that the demand for knee arthroplasty decreased in younger patients, and paralleled the general population requirements in older patients. The introduction of the use of methotrexate in the 1970s, and more recently the use of Tumour Necrosis Factor $\alpha$ has been attributed to milder forms of the disease and the slowing of radiographic deterioration [14].

\section{Aetiology}

In order to understand the aetiology of anterior knee pain it is vital to appreciate the biomechanical factors governing normal loading conditions. The weight distribution through the knee is governed by the mechanical axis of the lower limb. Usually this passes through or just medial to the centre of the knee joint in the coronal plane. Deviation of the mechanical axis away from this leads to increased contact stresses at the joint surface. 
Morrison et al 1970 [15] showed that the mean maximum joint force at the knee was 3.03 times body weight (range 2.06-4.0) during normal gait cycle. During stance phase the centre of pressure passed through the medial side of the joint, indicating that most of the joint force is centred there.

Understanding the movement of the tibiofemoral joint is aided by magnetic resonance imaging (MRI) studies [16]. As the knee flexes the femoral condyles roll backwards and slide on the tibia. This mechanism, along with the increasing arc of curvature of the posterior aspect of the femoral condyles, allows increased flexion of the knee. Dynamic studies have shown the lateral femoral condyle to have increased backward movement of the contact area relative to the medial femoral condyle. This in effect produces rotation of the tibia internally as the knee flexes.

The patella forms part of the extensor mechanism of the knee and has thick articular cartilage to withstand the joint reaction force. It allows an increased lever arm of the extensor mechanism which enhances its strength. The medial and lateral articular facets enter the trochlear groove of the femur at $20^{\circ}$ of flexion and maximum contact is achieved with the femur at $45^{\circ}$. The peak contact pressures within the patellofemoral joint occur between $60^{\circ}$ and $90^{\circ}$ of flexion. This range corresponds to the areas where degenerative lesions are most commonly seen. In the simple action of rising from a chair the force going through the patellar tendon can reach 3.6 times body weight [17]. The joint reaction force can reach $385 \mathrm{~N}$ during walking, and reach $5972 \mathrm{~N}$ when landing from a jump [18].

Hip pathology, from childhood through to adulthood may present as femoral or knee pain and must be eliminated as a cause of symptoms, as does neurological pain due to proximal nerve lesions. Pain is transmitted from nociceptors distributed around the knee. Articular cartilage does not contain nerve endings, therefore damage to this layer is not a direct source of pain. Nociceptor fibres, including those transmitting substance $P$, have been found to be widely distributed in the soft tissues around the knee joint [19], including the retinaculum, synovium and fat pad. Higher concentrations have been found immediately surrounding blood vessels, suggesting a role in vascular tone and extravasation of fluid leading swelling and effusion. Biedert et al 1992 [20] found increased concentration of type IVa nerve fibres were found in the retinaculum, patellar ligament and pes anserinus. Gronsblad 1985 [21] reported the presence of Substance P-immunofluorescent nerves and enkephalinimmunofluorescent nerves in the synovial membrane and the menisci. Dye 1998 [22] underwent arthroscopy of his own knees while conscious, and identified anterior synovium, fat pad and joint capsule as the most sensitive areas to pain. The insertion of the cruciate tendons provokes some pain, as did peripheral meniscus. The articular surface of the patella did not evoke significant discomfort. Hochmann et 2011 [7] looked at the contribution of neuropathic pain in patients with chronic knee pain. Having excluded those with pre-existing neurological conditions, $19 \%$ reported symptoms consistent with neuropathic pain.

Arthritic pain is commonly described as a dull ache, which is made worse on movement after a period of rest. While the loss of articular cartilage in osteoarthritis is not a direct source of pain, the resultant changes to the subchondral bone show radiological and 
histological changes. The loss of the articular cartilage changes the stresses on the underlying subchondral bone, which itself has pain receptors. Subchondral bone may react to increasing shearing forces by becoming reactive and sclerotic [23]. Bone scintigraphy studies have shown an increase in delayed phase tracer in the tibiofemoral compartment in of patients reporting knee pain [24]. This is likely to represent increased metabolic bone turnover in response to the changing physical stress environment of the bone. The increased tracer uptake has been shown to have a sixfold increased risk of progression to OA [25].

The increasing use of MRI in evaluating knee pain has allowed detection of histological changes not previously seen on plain radiographs. Bone marrow oedema, or bone marrow lesions (BML), appear on MRI as increased signal on fat suppressed T2 weighted images. The presence of BMLs has been associated with an increase in reported pain, and have a strong association with progression to radiographic changes of OA. This may help explain the degree of symptoms in patients with only mild radiographic changes associated with arthritis. McAlindon 1992 [10] showed that only 54\% of a cohort of patients over 55 years old with knee pain had radiographic evidence of OA. Conversely $17 \%$ of asymptomatic patients had radiographic changes consistent with OA, suggesting that radiographic changes alone do not fully explain the symptoms reported. In the presence of OA, Hill 2001 et al [26] found that the degree of synovial thickening and presence of an effusion correlated with the level of pain reported. It is also likely that individuals differ in perception of pain. Valdes et al 2011 [27] has reported an amino acid variant in the TRPV1 gene is associated with lower pain thresholds in radiographic OA.

Rheumatoid arthritis (RA) is a destructive inflammatory arthropathy that affects the small joints of the hands and feet, along with the large joints. An autoimmune reaction targets the synovial lining of the joint, leading to destruction of the cartilage and changes to the soft tissue structures around the knee causing deformity. The altered biomechanics of the joint accelerate the degenerative changes of the cartilage. Without appropriate pharmacological treatment or surgical intervention it can lead to profound disability and pain. The evolution of disease modifying anti-rheumatoid drugs (DMARDs) has had a significant effect on the number of patients requiring surgery [13].

Gout and pseudogout are caused by the deposition of crystals within the joint. The crystals induce the release of mediators resulting in inflammation. Whilst the reaction is usually selflimiting, its course can be shortened with use of anti-inflammatory medication. There has been increased incidence in gout noted over the last three decades [28]. Although genetic factors feature, it is also thought to be related to diet, lifestyle and increased longevity. Gout is the result of deposition of monosodium urate crystals within the joint. Urate is produced in the metabolism of purine, and is excreted in urine. If serum urate levels are elevated' monosodium urate levels in the extracellular fluid become saturated and crystals develop.

Acute soft tissue injuries around the knee cause pain and a variable amount of swelling. The anterior cruciate ligament (ACL) is supplied by the middle genicular artery. Acute rupture of the ligament activates nociceptor fibres, and the ensuing haemarthrosis stretches the joint capsule. While the initial pain subsides, the consequence of the altered biomechanics of the 
knee leads to chronic symptoms. Lohmander 2004 [29] reported that 12 years after injury of the ACL in young female athletes $75 \%$ reported significant knee pain and $42 \%$ had symptomatic radiographic knee osteoarthritis, whether a reconstruction was carried out or not.

Meniscal injuries can also be acutely painful at the time of injury. Proposed sources of pain include the resulting synovitis or distortion of the capsule from the meniscal tear. Mine et al 2000 [30] reported the presence of nociceptor fibres, mainly in the more vascular outer third (the red zone) and also at the anterior and posterior horns. This suggests an additional source of pain, although it does not fully explain the discomfort experienced from tears in the avascular inner third (white zone).

Like ACL injuries, meniscal injuries can lead to long term degenerative change. Berthiaume et al 2005 [31] used serial MRI to show that meniscal injury and volume loss was associated with an increased risk of degenerative change. Many people with symptomatic meniscal tears choose to have a partial or total meniscectomy. Roos et al 1998 [32] presented the results of a 21 year follow up study of patients post total open meniscectomy. The relative risk of developing tibiofemoral OA was 14.0 compared controls. Currently modern treatment algorithms have been to conserve healthy meniscus using a minimally invasive approach. Englund et 2004 [33] reported that even with limited resection the relative risk of radiological OA was 7.0 compared to matched controls, and 2.7 for symptomatic OA.

Anterior knee pain can occur in both children and adults. Both groups can be difficult to treat and the exact diagnosis can be elusive. Whilst there are certain terms used to describe the mechanisms by which the pain occurs, there will be cases where a true diagnosis is never made. In the past the term chondromalacia patella has been used. This should be avoided as it falsely attributes a pathological process and may lead to incorrect treatment and affect patient expectations.

Wear of the articular cartilage in the patellofemoral joint is associated with chronic knee pain. Like the tibiofemoral compartment not all with radiographic features of arthritis have symptoms [10]. The cause of the articular wear can be mechanical or inflammatory in nature. The even distribution of load across the joint avoids stress concentration, the alignment of the patella is thought to contribute to acute and chronic symptoms. There is some anatomical variation in the shape of the joint [34], which may predispose the early degenerative change. Dye et al [35] reports a shift in thinking away from biomechanical malaligment of the patellofemoral as a cause of acute pain. Instead he proposed that inflammation of synovium and fat pad along with increased metabolic activity in subchondral bone have aetiological importance.

\section{Knee pain in children \& adolescents}

There are recognised pathological changes that are unique to the skeletally immature knee [36]. Overuse of the knee can result in pain that only resolves after a period of rest. This is due to stress injuries from repetitive use, usually because of increased demands placed on 
the knee. They are also more common during growth spurts. Once the knee has been afforded time to recover from such stress then the symptoms usually resolve. The junction of the patellar ligament at the tibial tuberosity is a common site for such pain. Termed Osgood Schlatter's disease, the pain is in response to repeated stress causing mild avulsion injuries. The natural history is one of spontaneous resolution before adulthood [37]. SindingLarsen-Johannson disease presents as pain at the distal pole of the patella and responds to a simple non-operative therapy like Osgood Schlatter similar to the treatment of Osgood Schlatter disease.

\subsection{Traction apophysitis}

Osgood-Schlatter and Sinding-Larsen-Johansson disease is commonly seen in the male 12-14 age group. Kneeling is painful in Osgood-Schlatter disease and the tibial tubercle is painful to palpation. There may also be a prominence which is visible on the XR knee lateral. In later stages the tubercle will have a fragmented appearance due to the separation of the chondroosseous fragments [1] Treatment is conservative with reduction in the usually very high level of activity and simple analgesics (NSAIDS) till the symptoms have subsided. Quadriceps strengthening is avoided as this increases stresses across tibial tuberosity. This usually resolves within one to two years although the bony tibial tubercle prominence may persist. If they remain symptomatic there may be an ossicle in the substance of the tendon with an associated bursa of which surgical removal can be curative.

Whereas Osgood-Schlatter disease affects the tibial tubercle Sinding-Larsen-Johansson disease affects the lower pole of the patella. Treatment is the same as that for OsgoodSchlatter, namely conservative.

Osteochondritis dissecans should be considered as a cause if tibiofemoral and anterior knee pain. Most commonly affecting the lateral aspect of the medial femoral condyle, its cause has not been established although repeated trauma and vascular insult are likely to play a role [37]. A multipartite patella may also cause anterior knee pain, and may be detected on plain radiograph. Saupe 1943 [38] described three types: inferior pole, lateral patellar margin, and superolateral pole. Pain usually originates from the junction of the main body of the patella and the fragment.

Idiopathic anterior knee pain in the adolescent is difficult to treat. The cause has not been ascertained and prescribed treatments not always successful. The term chondromalacia patella has occasionally been used in describing such a problem, however this should be avoided as the term describes chondral changes that are not always present in anterior knee pain. Nimon et al [39] carried out a 14-20 year follow up on idiopathic anterior knee pain and determined that no operative intervention provided a better long term outcome than non-operative treatment.

Tumours should be considered as a cause of continuing knee pain, especially when night pain is present. There may also be localised swelling and skin changes. Malignant primary 
tumours are less common, if there is any doubt as to the diagnosis then at least plain radiographs in two planes should be sought.

\subsection{Classification}

Knee pain can present as an acute episode or a more chronic course. It is usually described by the causative pathology. The common causes of knee pain change with age of the patient, in children soft tissue and tendon causes are more common while in older adults arthritis is more prevalent.

Knee pain can be classified according to the affected compartments or structures. Anatomically the knee is divided into the tibiofemoral joint, medial and lateral, and its associated soft tissue structures. The patellofemoral joint is described separately, along with the associated tendinous structures that form the extensor mechanism of the knee.

The tibiofemoral joint contains the weight bearing surfaces of the femur and the tibia. The medial and lateral menisci are interpositioned between the two and have a role in load distribution. The anterior and posterior cruciate ligaments and the medial and lateral collateral ligaments provide stability along with the contents of the posterolateral corner of the knee.

The anterior part of the knee includes the patella, which acts to increase the lever arm of the extensor mechanism of the knee. The quadriceps insert into the proximal pole and the patellar ligament (also referred to as patellar tendon) links the inferior pole with the tibial tuberosity. The infrapatellar far pad lies posterior to the patellar tendon and is richly innervated.

Acute episodes may be caused by trauma to the bone, articular surface or soft tissues, but also include localised inflammation or minor injuries due to overuse. Chronic episodes often involve degenerative change to the articular surfaces. Arthritis can be idiopathic, or secondary to trauma. Inflammatory arthritis includes rheumatoid arthritis, gout, reactive arthritis and septic arthritis.

There is no broad consensus on classification of OA of the knee. Radiological findings have been described using the definitions of Kellgren and Lawrence. This has been widely used, including the World Health Organisation (WHO) epidemiological studies. Schiphof 1998 [40] assessed 25 different classification criteria of osteoarthritis which could be broken down into radiological, clinical, and both radiological and clinical criteria. One reason why a consensus does not exist is that radiographic findings may not relate to symptoms.

Post 2005 [41] divided anterior knee pain (AKP) into constant pain not activity related, sharp intermittent pain, and activity related pain. The constant pain may be neurological in nature, whether from sensory nerve pathology or autonomic nerves. Also included is pain reported for perceived secondary gain. Sharp intermittent pain usually relates to unstable structures within the knee or loose bodies. Activity related pain is divided into soft tissue overload with normal patellar alignment, or articular tissue overload and degenerate changes of the chondral surface. 


\subsection{Knee pain classification}

\begin{tabular}{|l|c|c|}
\hline Anterior knee pain & Pain type & Distinct/obscure \\
\hline Patellar tendonitis & Activity related & Distinct \\
\hline Bipartitite Patella & $\begin{array}{c}\text { Intermittent and } \\
\text { sharp }\end{array}$ & Distinct \\
\hline $\begin{array}{l}\text { Osgood-Schlatter/Sinding-Larsen- } \\
\text { Johansson syndrome }\end{array}$ & Activity related & Distinct \\
\hline Osteochondritis Dissecans & Activity related & Distinct \\
\hline Synovial Impingement & $\begin{array}{c}\text { Intermittent and } \\
\text { sharp }\end{array}$ & Distinct \\
\hline Chondromalacia patellae & $\begin{array}{c}\text { Intermittent and } \\
\text { sharp }\end{array}$ & Obscure \\
\hline Patellofemoral osteoarthritis & Constant & Distinct \\
\hline Patellar maltracking & Activity related & Obscure \\
\hline Excessive lateral pressure syndrome & Activity related & Obscure \\
\hline Tibiofemoral knee pain & \multicolumn{2}{|c|}{} \\
\hline Tibiofemoral OA & Constant & \\
\hline Meniscal tears & $\begin{array}{c}\text { Intermittent and } \\
\text { sharp }\end{array}$ & \\
\hline Ligamentous injuries & Activity related & \\
\hline
\end{tabular}

Table 1. Knee pain classification

\section{Diagnosis and management}

\subsection{Diagnosis}

Whilst history, examination and standard radiographic imaging are important in the determination of the aetiology of knee pain, newer imaging techniques and pain maps can also supplement the investigation of the causes of knee pain. This section will summarise pertinent symptoms and signs in the assessment of knee pain as well as describe advances in pain mapping and imaging.

\subsection{Patient history}

Several points in the history can yield very important diagnostic information about the cause of knee pain. Pain on sitting or climbing/descending stairs is often secondary to patellofemoral aetiology. Pain on squatting often with symptoms of locking or clicking usually relate to a meniscal tear. ACL tears are often described as a sudden 'pop' with immediate swelling and an inability to continue the activity that preceded it. This is a pivoting injury and can be without significant contact. Conversely the same symptoms with a significant contact force may suggest a collateral ligament tear. In both cases acute 
swelling will occur. A blow to the anterior tibia such as a dashboard or snowboarding injury can result in a Posterior cruciate ligament (PCL) tear. A sensation of an unstable knee or 'giving way' suggests ligamentous laxity or patellar instability. A history of sharp pain may be related to synovial impingement or loose bodies within the knee. Aching pain or pain after activity is thought to be related to inflammatory disorders whereas pain during activity is associated with structural abnormalities such as patellar subluxation or dislocation [42]. OA knee pain is typically insidious in onset and aggravated by movement and weight bearing and relieved by rest.

\subsection{Patient examination}

When examining a patient observation of their gait and stance can give valuable clues to their diagnosis. Quadriceps circumference if decreased indicates atrophy from inactivity. A palpable knee effusion is relatively non specific but can suggest a meniscal or ligamentous injury in the acute setting or arthritis in the chronic setting. Joint line tenderness indicates a meniscal tear which can also manifest as a block to full extension if big enough (such as a bucket handle tear). Loose bodies can also cause a locked knee.

Patella apprehension tested by pushing the patella laterally at $20-30^{\circ}$ of flexion indicates subluxation or dislocation. Patella grind and crepitus signify patellofemoral pathology. The Q-angle as measured from the angle between a line from the anterior superior iliac spine to the centre of the patella and a line from the centre of the patella to the tibial tubercle is increased with patella malalignment representing the degree of valgus translational force exerted upon the patella with quadriceps contraction.

Varus and valgus stress testing will confirm a lateral collateral ligament (LCL) and medial collateral ligament (MCL) injury respectively. Lachman test will be more sensitive than anterior draw for an anterior cruciate ligament (ACL) injury. A pivot shift test with the knee flexed at $20-30^{\circ}$ and internal rotation with valgus stress can also signify an ACL tear. The pivot shift however can only be done under anaesthesia. A posterior draw test is sensitive for a PCL injury although care must be taken in misinterpreting a positive posterior draw test as an ACL rupture if the tibia can be translated anteriorly in the anterior draw test.

To test for a posterolateral corner injury an assistant is needed. With the knees stabilised the feet are externally rotated and the thigh foot angle is compared at both 30 and $90^{\circ}$ of knee flexion. If there is asymmetry of greater than $15^{\circ}$ at $30^{\circ}$ flexion only this represents an isolated posterolateral corner (PLC) injury. If asymmetry is present at both 30 and $90^{\circ}$ knee flexion this represents a combined PCL-PLC injury.

\subsection{Radiological imaging}

Conventional radiography of the knee comprises a standard anteroposterior view and a lateral view. The lateral view allows assessment of the vertical position of the patella such as in patella alta (associated with lateral patella dislocation/subluxation, chondromalacia, patella ligament rupture, and Sinding-Larsen-Johansson) and baja (quadriceps tendon 
rupture, neuromuscular disorders and achondroplasia). Measurement of patellar tilt on the lateral radiograph with the knee if full extension is more sensitive for patellofemoral joint (PFJ) pain and prior dislocation than measures on the axial view [43]. The axial will show PFJ static alignment. The Insall ratio [44] is used to determine relative patellar height. The congruence and sulcus angles are indices of patellar subluxation. Trochlear depth may be measured using the sulcus angle. The congruence angle is a measure of lateral patellar displacement. The lateral patellofemoral angle on the axial view is a measure of patellar tilt. Abnormalities of these measurements may represent mal-alignment although they may be normal in asymptomatic patients when measured in mild degrees of flexion [45]. Computed tomography (CT) allows axial plane evaluation of the PFJ relationships in varying degrees of flexion for detecting malalignment. Imaging over the range of $5-30^{\circ}$ of flexion is the most useful for discriminating between normal and abnormal patellar tracking since beyond $30^{\circ}$ the patella centralizes in the trochlear groove. Rapid techniques able to capture images during active knee motion are described. This is now performed using helical CT which allows continuous rotation of the X-Ray tube and detectors during active joint motion. From knee extension to $45^{\circ}$ flexion 1 image a second is captured resulting in a total scan time of 10 seconds [46].

Magnetic resonance imaging (MRI) can visualize the components of the extensor mechanism and show lesions of articular cartilage and menisci, plicae and osteoarthritic change. Static MR imaging axial sections are obtained with the knee imaged at 4-8 different angle positions. This can be performed using any MR sequence without motion artifact. The disadvantage however is that joint kinematics and muscle contraction effects cannot be assessed. Kinematic MRI using motion triggered or ultrafast imaging techniques can be used to assess the contribution of associated soft-tissue structures to PFJ function with ultrafast imaging being less dependent on patient cooperation and can be performed even with severe patellar pain yielding fewer motion artifacts and better image quality. Kinematic MR imaging is also superior to axial radiographs in assessing patellar realignment surgery as significant differences between pre and post operative measurements are better demonstrated [47]. Overall MR imaging (especially ultrafast sequences) is felt to be superior to CT imaging in evaluation patellofemoral tracking. Muhle et al [46] feel that it should always be used in such cases in preference to CT unless the patient has contraindications to MR imaging. Different MR sequences are appropriate for imaging specific pathologies. Synovitis is best assessed by gadolinium enhanced MR sequences. Joint effusion is best detected on fat suppressed PD or T2 weighted FSE sequences. BML are best visualized on fat suppressed T2 weighted or STIR images. Menisci are typically visualized on sagittal PD $(\mathrm{TE}<20)$ FSE sequences and Ligaments on PD or T2 weighted FSE sequences.

\subsection{Functional MRI}

How the brain processes noxious stimuli in different disease states has been investigated using functional MRI (fMRI). This is also to map the cortical location to which the pain is mapped. fMRI uses a fast MR imaging method for which the image intensity is sensitive to the variations in magnetic field created in the tissue by the presence of paramagnetic 
deoxyhaemoglobin [48]. An increase in blood flow due to increased metabolic activity while processing a stimulus leads to decreased deoxyhaemoglobin and a small increase in intensity. Even with a modern 3 Tesla scanner this is only $10 \%$ increase in signal. With such low sensitivity several measurements are needed in individual patients or multiple patient datasets. Results from fMRI investigations has revealed that central activation of the brain mediates pain during $\mathrm{OA}$ and that non steroidal anti inflammatory drugs (NSAIDs) therapies may be partially acting via a central mechanism [49]. Furthermore in a recent paper by Gwilym et al 2009 [50] suggested that in specific groups of patients addressing central components of central pain processing by fMRI may be important for the treatment of long term OA pain.

\subsection{PET}

Positron emission tomography (PET) can be used to create two or three dimensional images of blood flow or metabolic processes [48]. Studies have shown arthritic pain is also associated with areas of the brain implicated in affect averse conditioning and motivation as well as the normal pain perception areas. Such findings have important implications for the management of patients with long term OA.

The use of fMRI, Positron emission tomography (PET), Single-photon emission computed tomography (SPECT) and Magnetoencephalography (MEG) are novel imaging modalities that have helped unravel how central pain pathways in the perception of chronic pain function.

\subsection{Scintigraphy}

This is a diagnostic test used in nuclear medicine. Radioisotopes taken internally emit radiation that is captured by external detectors (gamma cameras) to form a two dimensional image. Radioisotopes such as technetium-99m-MDP are preferentially taken up by bone and the specificity can be increased by performing an indium 111-labeled white blood cell test combined with a Technetium-99m-MDP injection. Conditions that cause increased bone turnover or inflammation can be diagnosed.

As such bone scanning can be used to detect fractures within 24 hours of the injury. This initially presents as increased vascularity and delayed scans can show increased uptake for up to six months after a fracture. Similarly bone bruises or trabecular fractures cause increased vascularity and uptake and are often associated with ligamentous injuries which show as bone bruised patterns in the adjacent site of bone attachment. Bone scanning is sensitive at showing stress fractures. Osteoarthritis causes increased uptake of radio nucleotide around the articular surface of the bone because of increased bone turnover. Increased uptake on both sides of a joint is characteristic of osteoarthritis. Bone scanning can also be helpful in the diagnosis and monitoring of CRPS following treatment [51]. Osteomyelitis shows increased vascularity and increased uptake on a three phase bone scan. Similarly cellulitis can also be diagnosed on a bone scan. Scintigraphy presents physiological 
features whereas radiography, CT and MRI all give structural information. In recent years, however, MRI scanning has largely replaced bone scanning for the diagnosis of localised knee pain.

\subsection{USS}

Ultrasound (USS) is a diagnostic imaging technique in the context of orthopaedics to visualize muscles and tendons tendons, nerves, ligaments, soft tissue masses, and bone surfaces. Compared to CT and MRI, USS is relatively inexpensive, portable and safe as it does not use mutagenic ionising radiation [52]. It is however largely operator dependent, and is unable to evaluate bone and other structures.

Although USS can be used for detection of ligament ruptures and meniscal injuries within the knee [53,54], this role has largely been superseded with the use of MRI.

Ultrasound is ideally placed for the assessment of tendon lesions, particularly partial and complete quadriceps rupture and tendinitis of the patellar tendon [55]. USS can also evaluate muscle injury and differentiate between partial and complete tears [54]. It is also very valuable in differentiating between swellings around the knee such as popliteal cysts and other local swellings including aneurysm, nerve sheath tumour and ganglia [56].

Joint effusions and bursae are easily detected by ultrasound. Ultrasound guided aspiration of bursitis can be carried out if necessary.

\subsection{Arthroscopy}

Arthroscopy is useful for direct visualization of bone, cartilage and soft tissue. Despite advances in non-invasive imaging techniques it is still considered the most reliable method for assessing patellar position and tracking. CT and MRI however, still provide useful augments.

\subsection{Bone marrow lesions and osteoarthritis progression}

Bone scans of patients with OA have shown increased uptake of tracer in the subchondral bone signifying increased turnover [25]. This was found to be associated with increased joint pain and increased risk of progression to radiographic OA. Similarly this has been demonstrated on MRI as increased signal in the marrow on fat suppressed T2 weighted images and has been dubbed bone marrow lesions [57]. Histological these manifest as bone marrow necrosis, trabecular abnormalities and bone marrow fibrosis and oedema [58]. It has also been reported that in patients with radiographic OA those who also had BM oedema lesions more often had knee pain than those without [59]. BMLs are localized to the subchondral bone. As the subchondral bone is innervated BML are thought to be a potential source of pain. Recent longitudinal studies have demonstrated that bone marrow lesions powerfully predict risk of local structural deterioration in OA knees. The risk of medial progression of OA within the knee was increased sixfold if there were medial BMLs. Similarly the risk was increased laterally if there were lesions laterally. Furthermore varus 
knees had a very high prevalence of medial bone marrow lesions whereas valgus knees had preferential lateral lesions. What is not clear however is whether the limb malalignment itself causes the progression of OA and development of the BML or whether OA progression is a consequence of the presence of BMLs [25]. Tanamas et al [60] found that the relationship between BML presence and cartilage change was independent of tibiofemoral alignment. Furthermore as there is no treatment for BMLs currently there is no justification for the routine use of MRI in assessing OA patients particularly as it is not clear if the lesions themselves cause structural damage or are a consequence of malalignment.

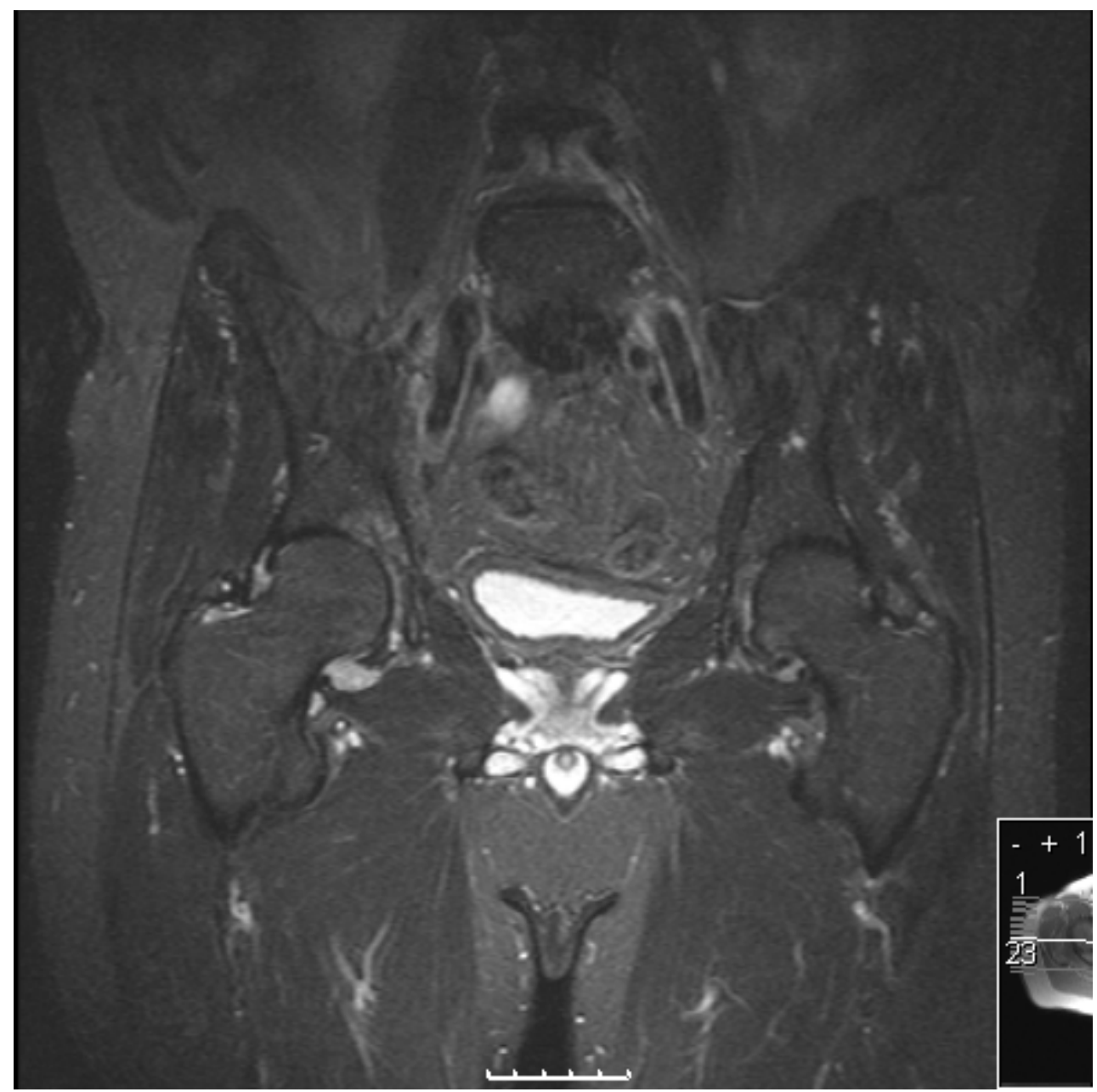

Figure 1. Coronal STIR MRI showing subchondral bone oedema and synovitis

Until recently there has been much focus on articular cartilage in assessing OA severity. There is conflicting data about whether cartilage damage correlates with pain. And recent 
studies have tied to shift the emphasis to other pathological events such as BML and synovial effusion/synovitis in understanding the cause of OA pain. The clinical implication of BML is not clear. It is present in $78 \%$ of patients with knee OA with pain and in $30 \%$ of patients in knee OA without pain [59]. BML is not pathognomonic of knee OA but its association is intriguing and not fully understood. This only highlights the need for more research into the matter. It is becoming more evident that pain from OA is multifactorial and that research into such new fields will help predict disease progression and alter improve treatments.

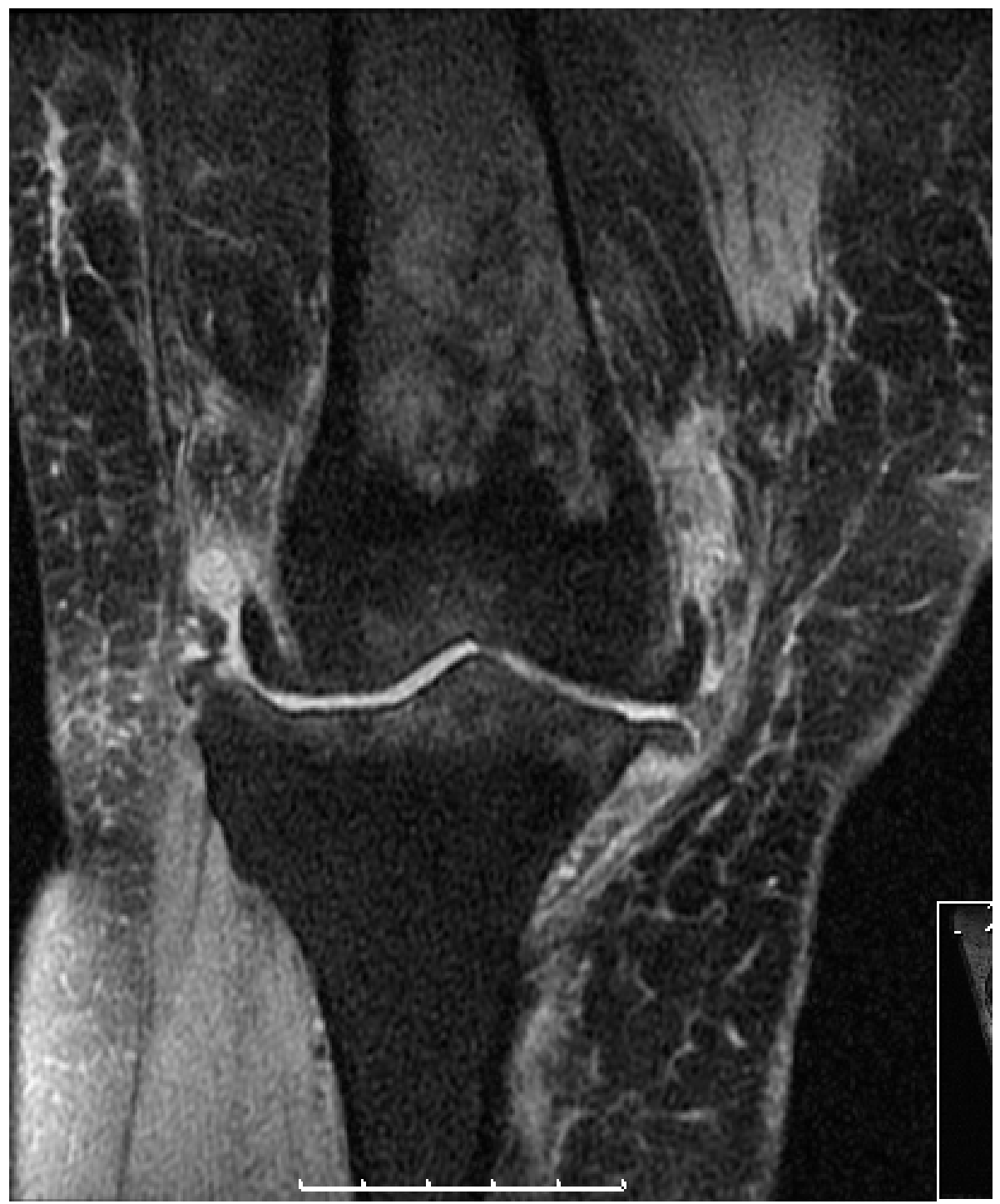




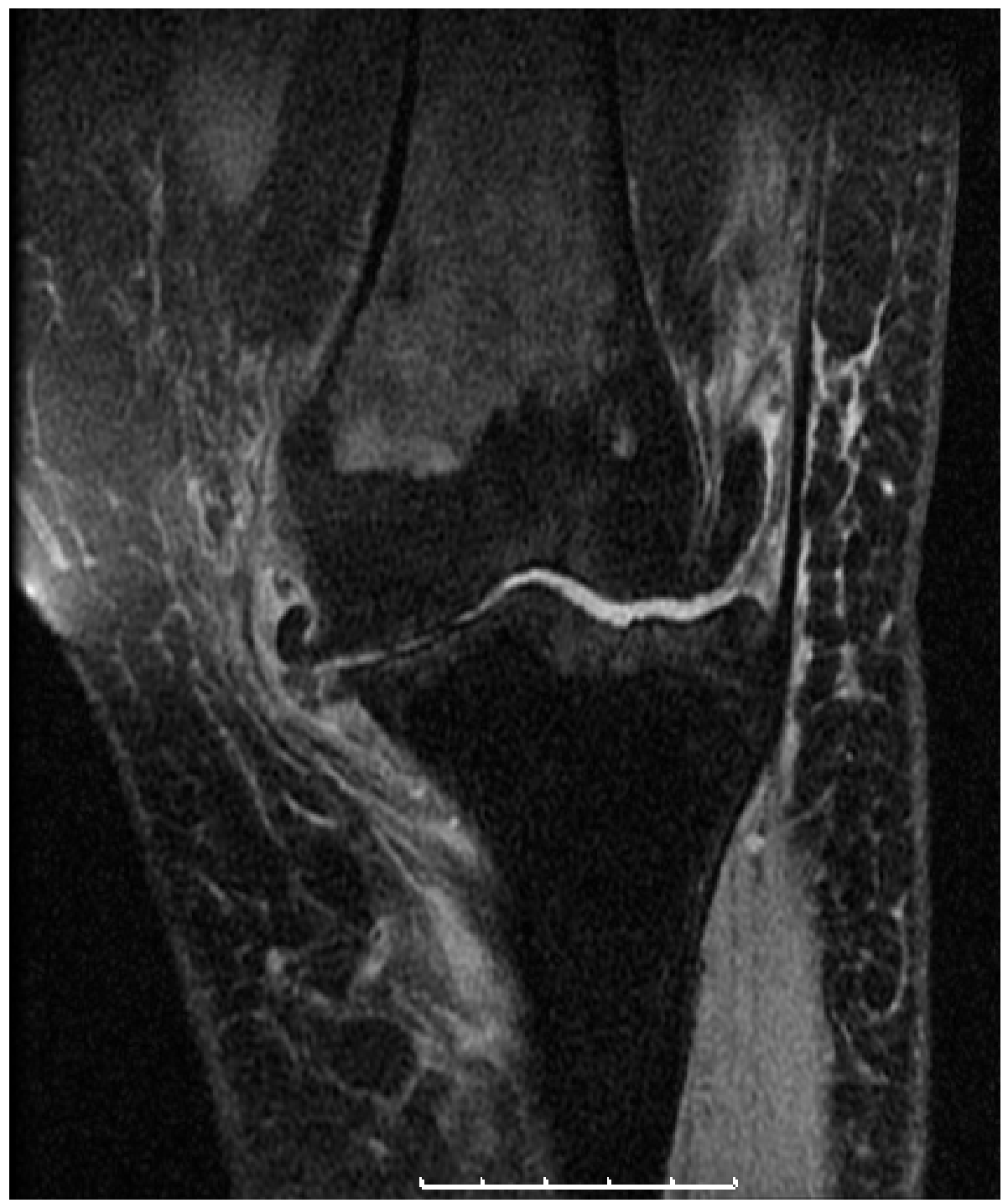

Figure 2. Coronal PD FS images showing severe OA with complete cartilage loss and subarticular tibial plateau bone oedema

\section{Anterior knee pain}

\subsection{Patella tendon tendinitis}

Also known as Jumpers knee this is tendinitis at the tendon-bone interface of the lower pole of the patella. This manifests as point tenderness of the lower pole of the patella. The decline squat test will be positive. This can also be confirmed on USS or MRI. The treatment is 
initially non-operative with physiotherapy and oral anti-inflammatory medications. Steroid injection is contraindicated since this may cause tendon rupture. Cases refractory to conservative management can be managed operatively to try and induce tendon healing by inflicting surgical trauma at the degenerative site [1]. This can vary from simply scrapping away at the site of degeneration to stripping of the deeper half of the tendon if it is affected to drilling the distal pole.

\subsection{Bipartite patella}

Common in childhood and usually bilateral. The most common site is the superolateral corner and this is the site most likely to be symptomatic. The most effective surgical treatment is simply excision of the fragment

\subsection{Osteochondritis dissecans}

This presents with pain and crepitus in knee extension against a load. Giving-way can be a result of pain inhibition. Plain $\mathrm{XR}$ including $30^{\circ}$ skyline view will yield a diagnosis. Classically the majority of lesions are found in the lateral aspect of the medial femoral condyle followed by the lateral femoral condyle and patella. Most commonly seen at the medial patellar facet. Differentiation from chondromalacia patellae may be difficult but chondromalacia tends to be confined to the overlying cartilage whereas osteochondritis involves a fragment of bone. There is a high incidence of contralateral limb involvement $15 \%-30 \%$ [61] and so bilateral knee XR is recommended. Subchondral sclerosis can indicate loosening of the fragment within the crater. CT and MRI will confirm it and show whether separation has occurred. The presence of homogenous high signal beneath the fragment of greater than $5 \mathrm{~mm}$ in diameter may represent an unstable lesion [62].

Arthroscopy will stage the lesion [1]:

1. Intact lesion with no break in articular cartilage

2. Separated lesion with no break in articular cartilage but beneath the intact layer the bone has separated

3. A trapdoor lesion in which the fragment of osteochondritis dissecans is partially detached

4. Detached loose body.

Stage 1 can be managed with activity modification and cessation of high impact sports especially in patients under 14 who would be expected to heal (the presence of an open physis is encouraging). Separated lesions can be stabilized with biodegradable pins or osteochondral bone pegs. Stage 3 and 4 should be excised leaving stable vertical edges to the crater and microfracture to induce healing within the defect with fibrocartilage.

\subsection{Synovial impingement}

Pathological plicae are difficult to diagnose as they are a normal finding. Superior, medial and inferior plicae are most common [63]. Identification on arthroscopy does not necessitate 
excision unless physical examination is abnormal. Pain is worse with knee flexion and relived by extension. There may be a thickened palpable cord [64]. Usually the medial plica. XRs are normal. MRI will identify it but will not necessarily determine if it is pathological. With stronger magnets for MRI this may change however as wear on the medial femoral condyle secondary to a thickened medial plica is identified. Conservative management with activity modification, steroid injections into the plica and physiotherapy is initial treatment followed by arthroscopic excision if necessary.

Hoffa syndrome presents with swelling in the region of the fat pad. Diagnosed with the Hoffa maneuver of applying compression either side of the patella tendon while extending the knee. The test is positive if it elicits pain or apprehension. Diagnosis can be confirmed on MRI as high signal content consistent with irritation of the fat pad [65]. As before treatment is with activity modification then debridement if necessary.

\subsection{Chondromalacia}

Degenerative condition of the articular cartilage not often seen in skeletally immature. XR are often normal or may show joint space loss if cartilage loss is extensive. Diagnosed on MRI and Arthroscopy. T2 weighted images with fat saturation or fast spin echo intermediate weighted MRI demonstrate good contrast at the interfaces between cartilage and subchondral bone and between cartilage and joint fluid [66]. Usually imaged in the axial plane identifying potential cartilage defects and internal derangement of the cartilage layer before gross morphological cartilage loss. In idiopathic cases with limited damage unrelated to patella subluxation and/or tilt it is acceptable to improve the articular surface arthroscopically. This reduces the amount of debris in the joint and decreases release of proteolytic enzymes and decreases synovitis and delaying osteoarthritis onset [67]. The malacic areas are excised and the subchondral bone is drilled to encourage repair with fibrocartilage. This however will not withstand the large loads and sheer forces hyaline cartilage can. Osteochondral grafting in the form of mosaicplasty can be successful for defects on the femoral condyles but not the undersurface of the patella.

\subsection{Patellofemoral osteoarthritis}

The standard radiographic features of OA are joint space loss, subchondral sclerosis, cysts and osteophytes. Such radiographic features are also visible on MRI.

\subsection{Patellar maltracking}

Treatment is dependant differentiating between patients with normal and abnormal anatomy. When examining close attention should be paid to any malalignment including genu valgum, external torsion of the tibia and femoral anteversion which may be associated subluxation or patellofemoral malalignment [68].

Imaging of the patellofemoral instability patient includes weight bearing $\mathrm{AP}, 45^{\circ} \mathrm{PA}$ flexion and lateral and axial views. These plain XR provide information about osteochondral 
fractures patellar height, morphology of the trochlear groove, patellar tilt and sulcus angle. Beyond $45^{\circ}$ flexion most patella engage fully with the trochlear groove. As such most imaging studies of the PF tracking should focus on $30-45^{\circ}$ flexion.

CT can be helpful in planning a realignment procedure if necessary. MRI will provide information regarding the medial retinacular structures (medial patellofemoral ligament (MPFL) injured in as many as $94-100 \%$ of acute patella dislocations [69]. MPFL is extracapsular and can be missed on arthroscopy which is important as MPFL repair is being increasingly advocated to prevent recurrent dislocation, chondral damage and ligament injuries, particularly in cases of acute patella dislocation. Quinn et al [70], described a triad of injuries on MRI consisting of impaction injury to the lateral femoral condyle, osteochondral defect (OCD) of the medial patellar facet and injury of the medial patellar retinaculum. As patellar dislocation can be transient MRI findings provide valuable clues to the pathology and can be the first indication of the diagnosis.

Skyline views provide only static images from which the patellar dynamics cannot be understood. It is important to delineate the pattern of maltracking in before considering management options.

Lateral subluxation in extension is demonstrated clinically by extending the knee against gravity. As the knee extends from $20^{\circ}$ flexion to extension the patella can be observed to kick laterally and can be accompanied by crepitus and pain as the patella disengages from a position of stability in the trochlear groove. This is commonly a problem of recurrent dislocations in childhood. The principal cause is malalignment of the quadriceps. This can be managed with physiotherapy or distal realignment of the tibial tubercle by $1.5 \mathrm{~cm}+/-$ proximal medial advancement of the patellofemoral ligament and lower fibres of vastus medialis. Lateral release and inner range quadriceps exercises are not appropriate.

Conversely maltracking in flexion is easier to see radiologically on skyline views but more difficult to examine for clinically beyond symptoms of instability and pain. With pure subluxation in flexion chondral damage is mild and a simple lateral release will often suffice [1]. However with combined subluxation and tilt the lateral facet is overloaded and the chondral damage can be more severe. Subsequently the threshold for performing a distal realignment is lower.

CT is a very useful imaging modality to study patella tilt and subluxation. Arthroscopy through anterolateral and superolateral portals will allow assessment of patellar tracking between $0-90^{\circ}$ and also allow the clinician to observe overhang of the lateral facet.

Chronic patellofemoral instability nonoperative treatment focuses on regaining strength in the quadriceps mechanism and hip abductors. Traditionally for acute dislocation the knee was immobilized in cylinder cast or cricket pad splint. Others prefer early motion controlled with lateral buttress bracing. Operative management in the acute setting is indicated for osteochondral fragment repair or removal. It may also be indicated for repair or reconstruction of the medial retinacular structures. 


\subsection{Excessive lateral pressure syndrome}

Lateral patellar tilt is dominant with little or no subluxation. Imaging shows cartilage loss, sclerosis and cystic change of the lateral patella and trochlear. Dynamic studies show lateral tilt with increasing flexion.

\subsection{Patellectomy}

Indicated when the articular surface of the patella is so badly damaged over a wide area that it cannot be salvaged and an otherwise relatively normal knee. The patient should rehabilitate in a hinged knee brace back to their activities of daily living but will not be competitive in sports. Quadriceps wasting is inevitable and will not exceed $70 \%$ of normal despite extensive rehabilitation. Complications include rupture, subluxation and pain.

\subsection{Complex regional pain syndrome}

Complex regional pain syndrome (CRPS) manifests as severe pain, swelling and changes in the skin. It is often associated with autonomic dysregulation and can lead to functional loss, impairment and disability. Clinically there must be an initial noxious event that leads to continuing pain, allodynia or hyperalgesia with evidence of oedema and changes in skin blood flow in the area of pain. This must all be in the absence of another condition that could account for such symptoms.

Sweat testing can help in the diagnosis of CRPS. A trial of sympathetic block can also be useful given the aetiology of the condition. Conventional radiographs can also be useful in showing the resultant patchy osteoporosis although a three phase technetium bone scan can detect these changes sooner and will show higher bone density with successful treatment. Nerve Conduction Studies (NCS) are important tests in CRPS as they are very reliable for detecting nerve injury (causalgia).

Treatment is multidisciplinary and involves physiotherapy together with drug treatment such as antidepressants and gamma-aminobutyric acid (GABA) analogues.

\subsection{Pain in OA}

Pain is the most disabling symptom of OA. Previous work has focused on articular cartilage as the cause although we cartilage is not innervated with nerve fibres. With the advent of widespread MRI use we are no able to investigate the whole joint as an organ to identify the structures that cause pain such that $\mathrm{w}$ may develop targeted therapies. In a systematic review by Yusuf et al [71] there was a moderate level of evidence for a positive association for BML and effusion/synovitis with pain in OA. There was limited evidence for an association for knee ligamentous abnormalities and no association with osteophytes and and subchondral cysts. There was conflicting evidence for cartilage defects, meniscal lesions and bone attrition. 


\subsection{Physiotherapy management of patellofemoral pain}

The aims of interventions described are to improve patellar tracking and reduce abnormal stress on the PFJ structures.

Patients respond well to a home exercise program initiated by and regularly followed up with dedicated physiotherapists. General quadriceps exercises are most commonly employed together with specific vastus medialis obliquus (VMO) targeted training. VMO delay or reduction in relation to VL is thought to contribute to lateral patellar tracking. Quadriceps training is the basis of conservative management and studies indicate at least a moderate reduction in pain when performing such exercises including weight bearing and non-weight bearing exercises [72]. Witvrouw et al [73] studied the effects of open kinetic chain exercises (i.e. without weightbearing such as seated knee extension) versus closed chain exercises (such as leg press) in patients with anterior knee pain. Closed chain exercises produced less pain and better functional improvement although both exercises were beneficial.

Maximum compressive load is generated $90^{\circ}$ flexion in both types of exercises [74] but open kinetic chain exercises produce higher loads from $45^{\circ}$ to full extension. Escamilla et al [75] showed that closed chain exercises produced greatest quads activity at near full knee flexion with more activity in the vasti muscle whereas open chain exercises produced greatest quads activity near full extension with more rectus femoris activation. Further studies [76] confirmed greater VMO activity with closed chain exercises. Whilst closed chain exercises seem to confer more advantages not all patients will tolerate them as well as open chain. It is worth also noting that eccentric strengthening (muscle contraction while lengthening e.g. walking down stairs) produces the highest muscle strains and so may be recommended for the later stages of rehabilitation. Flexibility is important in eccentric loading to help absorb energy [41] and so patients undergoing rehabilitation for anterior knee pain will benefit from hip, quadriceps, hamstring and gastrocnemius stretching.

While an increased $Q$ angle is thought to predispose the patella to excessive lateral tracking this has been disputed in recent literature [72]. This may be related to the static nature of a $Q$ angle measurement that does not reflect real life kinematics. Souza et al et al [77] described the use of a dynamic $Q$ angle and theorized that femoral adduction and internal rotation relative to the pelvis impart a valgus knee force and stress lateral PFJ structures. This has been confirmed on kinematic MRI and has justified the use of targeted hip exercises in treating patients with PFJ pain. Results from studies indicate that those who participated in exercise programs targeting hip abductors and external rotators reported at least a moderate reduction in pain (72) Faulty foot mechanics can also increase the dynamic $Q$ angle [78] and has led to the use of foot orthosis for the treatment of PFJ pain with moderate success [79].

In combination with quadriceps exercise patellar taping is effective. Taping involves applying medially directed adhesive tape to glide, tilt and rotate to the patella to increase patellofemoral contact and reduce pain. Studies have reported a strong reduction in pain [80]. Whether this effect is due to improved patellar alignment or better proprioceptive awareness and neuromuscular control is still debated. The evidence for patellar bracing is less convincing although some studies have shown a moderate improvement [81]. It may be that bracing shifts contact of the patella with the trochlea to areas of non-irritation. 
Whitelaw et al [82] found that $87 \%$ of patients with PF pain improved with a combination of physical therapy and NSAIDS and that $68 \%$ of those patients maintained that improvement at 16 months. Kannus et al [83] followed up patients for 7 years and found that almost three quarters of patients maintained subjective and functional recovery from 6 months to 7 years with quadriceps exercises, rest and NSAIDs.

Based on such high success rates patients with PFJ pain should be managed with nonoperative measures till such time that symptomatic improvement has reached an unacceptable plateau.

\section{OA management}

\subsection{Simple analgesics}

Current OA treatment guidelines recommend paracetamol as a first line treatment followed by a NSAID. NSAIDS are proven to be better at improving pain and function in osteoarthritis. The combination of paracetamol and ibuprofen is associated with significant improvements in pain relief, function and quality of life as compared to paracetamol use alone for both short and long term use [84] but at the expense of an increase in side effects, primarily GI (PPI) bleeding.

Adjuncts to simple analgesics include weight loss and activity modification and physiotherapy. Opioid analgesics (such as dermal patches) are also used. Beyond that intra articular HA and steroid injections are considered the third step in management followed by surgical referral.

\subsection{NSAIDs}

Trials show that selective Cox-2 inhibitors are less harmful to the lower GI tract than conventional NSAIDS prescribed together with a proton pump inhibitor gastro-intestinal since PPIs offer no protection to NSAID induced effects on the small bowel and colonic mucosa [85].

\subsection{Hyaluronic acid injection}

Hyaluronic acid (HA) injections are widely used in the medical management of knee OA. There are a variety of different products available on the market with differing molecular weights, origins, and viscosities. Opinion is divided about the efficacy of HA injections. A recent meta-analysis [86] confirmed a modest efficacy for knee OA just minimally exceeding clinical significance. HA was found to be effective at 4 weeks post injection and reach peak effectiveness at 8 weeks with a residually detectable effect at 24 weeks. When at its peak effectiveness it appeared to be more effective than acetaminophen and NSAIDS and comparable to Cox-2 inhibitors.

\subsection{Glucosamine}

Controversy exists on the efficacy of glucosamine in the symptomatic treatment of knee OA. Meta-analyses have provided conflicting results. As with HA, conflicting trial results may be 
due to the use of different formulations. A recent large multicentre double blinded trial testing glucosamine and chondroitin preparations (GAIT) found that overall, there were no significant differences between the glucosamine or chondroitin preparations tested and placebo within the subset of patients with mild knee pain either together or alone. There was a subset of patients with moderate-to-severe pain, in whom glucosamine combined with chondroitin sulfate provided statistically significant pain relief compared with placebo. However, because of the small size of this subgroup these findings should be considered preliminary and need to be confirmed in further studies.

\subsection{Biotherapy}

Preclinical studies have shown that nerve growth factor regulates the structure and function of responsive sensory neurons including small diameter nociceptive afferents [85]. Tanezumab, an antibody against nerve growth factor (NGF) was clinically trialled as the first biotherapy for knee OA. Early results were impressive for efficacy but trials were halted by the FDA because of an unexpected increase in joint prosthesis in the tanezumab group compared to the placebo group. Schnitzer et al 2011 [87] showed that repeated injections of tanezumab in patients with moderate to severe knee OA provided relief (45-60\% reduction of pain) and better function with a low incidence of side effects (parasthesia).

\subsection{Pharmacological treatment reference guide}

\begin{tabular}{|c|c|c|c|}
\hline Simple & Mode of action & Typical dose & $\begin{array}{l}\text { Precautions/Side } \\
\text { effects }\end{array}$ \\
\hline Paracetamol & $\begin{array}{l}\text { Not fully understood } \\
\text { - likely COX } \\
\text { inhibitor }\end{array}$ & 500mg-1G QDS & $\begin{array}{l}\text { Hepatotoxicity in } \\
\text { overdose }\end{array}$ \\
\hline NSAIDS & COX inhibitors & $\begin{array}{l}\text { Ibuprofen 400mg TDS } \\
\text { Naproxen 500mg BD }\end{array}$ & $\begin{array}{l}\text { Gastrointestinal and } \\
\text { renal adverse reactions }\end{array}$ \\
\hline \multicolumn{4}{|l|}{ Opiate alternatives } \\
\hline Codeine & $\begin{array}{l}\text { Acts on opioid } \\
\text { receptor }\end{array}$ & 15-60mg QDS & $\begin{array}{l}\text { Nausea. } \\
\text { Constipation. } \\
\text { Itching. } \\
\text { Dependence. } \\
\end{array}$ \\
\hline Tramadol & $\begin{array}{l}\text { Acts on opioid } \\
\text { receptor }\end{array}$ & 50-100mg QDS & $\begin{array}{l}\text { Nausea. Constipation. } \\
\text { Itching. Dependence. }\end{array}$ \\
\hline \multicolumn{4}{|l|}{ Supplements } \\
\hline Glucosamine/Chondroitin & $\begin{array}{l}\text { Unknown - possible } \\
\text { proteoglycan } \\
\text { synthesis/cartilage } \\
\text { protection }\end{array}$ & $1500 \mathrm{mg} / 1200 \mathrm{mg}$ & Generally safe \\
\hline Hyaluronic acid & Lubricant & Intra-articular injection & Generally safe \\
\hline
\end{tabular}

Table 2. Pharmacological treatment reference guide 


\subsection{Weight loss}

Weight loss is difficult to achieve and is perhaps even more difficult to maintain. Despite this it is frequently recommended for the treatment of OA. In a study by Bliddal et al [88] reported at 1 year patients allocated to a low fat high carbohydrate and protein diet had reduced pain in the knee due to OA. This result and others indicate that approximately $30 \%$ of obese patients with knee OA can achieve significant long term weight loss and improve their pain symptoms. Though studies have shown an improvement in pain levels there is yet no evidence in difference in function or disability. However as pain is the main problem of OA there is good justification for advocating weight loss initially.

\subsection{Knee arthroplasty}

Knee joint replacement is an effective and cost-effective intervention for people with advanced OA. There are however no clear indications for the use of knee joint replacements and it can be difficult to know when in the course of OA it is best to operate [89]. This is evidenced by the great variability in rates of knee joint replacement [90 Furthermore a minority of patients are made symptomatically worse by surgery. There is also the difficulty of there being a number of different approaches and prosthesis available and the decision on whether to perform a total knee replacement or unicompartmental replacement or even high tibial osteotomy for the young functionally demanding patient with unicompartmental disease.

Ultimately the decision to operate will depend on the best available research evidence in combination with the experience of the surgeon depending on the individual patient's needs and expectations.

\subsection{Anaesthesia for knee operations}

Most knee operations are now done under a general anaesthetic or a regional anaesthetic or a combination of the two. For arthroscopic procedures theses are usually performed as daycase procedures and so a quick general anaesthetic is generally the rule. This is augmented with a local anaesthetic (such as $10 \mathrm{mls} 0.5 \%$ bupivacaine) injected into the knee joint and port sites to help with post operative pain relief.

Anaesthesia for knee arthroplasty should provide stable intra-operative conditions and allow adequate postoperative analgesia to promote early mobilisation and better surgical outcomes.

Regional anaesthetic techniques can have several advantages over general anaesthesia for knee arthroplasty such as less intra- and post-operative blood loss.

\section{Common techniques}

\subsection{Spinal anaesthesia}

Patients will be awake for the procedure. There is a reduced risk of venous thromboembolism and reduced blood loss compared with general anaesthesia. Spinal 
anaesthesia provides early postoperative analgesia and can be supplemented by patient controlled analgesia (PCA).

\subsection{Epidural analgesia}

Provides very good pain relief which can be extended postoperatively. However it is dependent on adequate anaesthetic input and training of nursing staff. It has the added requirement of needing urinary catheterisation which may delay postoperative mobilisation. Patients need to be aware that they will feel a sensory and motor disturbance affecting both legs.

\subsection{Femoral and sciatic nerve blocks}

Do not provide surgical anaesthesia but rather good pain analgesia for to the first 12-24 hrs. They avoid the need for a urinary catheter in most patients and allow mobility in bed.

\subsection{Pain coping skills}

Patients with chronic pain often develop maladaptive thought patterns known as pain catastrophising and abnormal behavior patterns such as guarding or inactivity that contribute to physical and emotional suffering. In a study by Riddle et al [91] patients receiving psychologist directed pain coping skills training reported significantly greater reductions in pain severity and catastrophising as well as improved function. This provides preliminary evidence that such treatments may be efficient in reducing the number of patients unhappy with the results of knee arthroplasty.

\section{Conclusion}

The diagnosis and management of knee pain can be challenging. As with most aspects of medicine, a thorough history and careful examination will often lead to the correct diagnosis. Traditionally XR and USS imaging have been the most accessible to the clinician in confirming the diagnosis. With the increased availability of CT and MR it is now possible to investigate knee pain in much greater depth within a shorter period of time without resorting to invasive procedures. With the advances in imaging techniques, particularly in MRI, soft tissue knee pathologies can be much better delineated and appropriate treatment planned. This has transformed the diagnosis and management of anterior knee pain. Nevertheless an experienced physiotherapist is invaluable in treating patients with anterior knee pain with surgery reserved for when a plateau has been reached which is unacceptable for the patient. With the advent of more powerful MR scanners and more sophisticated sequences we can also begin to understand the pathology of knee OA better. Although undoubtedly BMLs have a role in knee OA their exact relationship to progression of knee $\mathrm{OA}$ and causality is not yet fully understood. No doubt this is an area of future research and development. The role of analgesics in OA has been proven. As have the surgical options 
predominantly in the way of arthroplasty, with good proven results. It is the interim steps in between that are not yet proven and need further research and development. The role of biotherapeutic agents whilst temporarily sidelined may become more important if initial discoveries hold true. We know that pain from OA is likely to be multifactorial and with the advent of functional MRI we can begin to understand the central processing of pain in chronic knee OA. This may yield yet more possibilities for the treatment of OA.

\section{Author details}

Sherif Hosny, W. McClatchie, Nidhi Sofat and Caroline B. Hing

St George's Hospital NHS Trust, Blackshaw Road, London, UK

\section{References}

[1] Jackson, A.M., Anterior Knee Pain. Journal of Bone and Joint Surgery British, 2001. 83B: p. 937-48.

[2] Cutbill, J.W., et al., Anterior knee pain: a review. Clinical journal of sport medicine, 1997. 7: p. $40-45$.

[3] Kellgren J, Lawrence J. Radiological assessment of osteoarthrosis. Ann rheum Dis 1957;16: 494-502

[4] Ahlbäck S. Osteoarthrosis of the knee: a radiographic investigation. Acta Radiol Stockholm 1968; (suppl 277):7-72.

[5] Symmons D, Mathers C, Pfleger B. Global burden of osteoarthritis in the year 2000. Geneva: World Health Organization; 2003.

[6] Dawson J, Linsell L, Zondervan K, Rose P, Randall T, Carr A, Fitzpatrick R. Epidemiology of hip and knee pain and its impact on overall health status in older adults. Rheumatology 2004;43:497-504

[7] Haq S, Davatchi F. Osteoarthritis of the knees in the COPCORD world. Int J Rheum Dis 2011;14:122-129

[8] Ingham S, Zhang W, Doherty S, McWilliams D, Muir K, Doherty M. Incident knee pain in the Nottingham community: a 12-year retrospective cohort study. Osteoarthritis Cartilage 2011;19:847-852

[9] Nguyen U, Zhang Y, Zhu Y, Niu J, Zhang B, Felson D. Increasing prevalence of knee pain and symptomatic knee osteoarthritis: Survey and cohort data. Ann Intern Med. 2011;155:725-732

[10] McAlindon T, Snow S, Cooper C, Dieppe P. Radiographic patterns of osteoarthritis of the knee joint in the community: the importance of the patellofemoral joint. Ann Rheum Dis 1992; 51:844-849

[11] Jarvholm B, From C,Lewold S, Malchau H, Vingard E. Incidence of surgically treated osteoarthritis in the hip and knee in male construction workers. Occup Environ Med 2008;65:275-278 
[12] Miranda H, Viikari-Juntura E, Martikainen R, Riihimaki H. A prospective study on knee pain and its risk factors. Osteoarthritis Cartilage 2002;10:623-630

[13] Louie G, Ward M. Changes in the rates of joint surgery among patients with rheumatoid arthritis in California, 1983-2007; Ann Rheum Dis 2010;69:868-871

[14] Fevang B, Lie S, Havelin L, Engesaeter L, Furnes O. Reduction in Orthopedic Surgery Among Patients With Chronic Inflammatory Joint Disease in Norway, 1994-2004. Arthritis Rheum 2007;57:529-32.

[15] Morrison J. The mechanics of the knee joint in relation to normal walking. J Biomechanics 1970;3:51-61

[16] Freeman M, Pinskerova V. The movement of the normal tibiofemoral joint. J Biomechanics 2005;38:197-208

[17] Amis A Farahmand F. Biomechanics masterclass: extensor mechanism of the knee. Curr Orthop 1996;10:102-109

[18] Feller JA, Amis A, Andrish J, Arendt E, Erasmus P, Powers C. Surgical biomechanics of the patellofemoral joint. Arthroscopy. 2007;23:542-53.

[19] Witonski, Wagrowska-Danielewicz M. Distribution of substance-P nerve fibres in the knee joint in patients with anterior knee pain syndrome. Knee Surg Sport Traumatol Arthrosc. 1999;7:177-83.

[20] Biedert R, Stauffer E, Friederich N. Occurrence of free nerve endings in the soft tissue of the knee joint: A histologic investigation. Am J Sports Med 1992;20:430-433

[21] Gronblad M, Korkala O, Liesi P, Karaharju E. Innervation of synovial membrane and meniscus. Acta Orthop Scand 1985;56:484-486

[22] Dye, S.F., The Pathophysiology of Patellofemoral Pain. Clinical Orthopaedics and Related Research, 2005. NA;(436): p. 100-110.

[23] Bassiouni H. Bone marrow lesions in the knee: the clinical conumndrum. Int J Rheum Dis 2010;13:196-202

[24] Kraus V, McDaniel G, Worrell T, Feng S, Vail T, Varju G, Coleman R. Association of bone scintigraphic abnormalities with knee malalignment and pain Ann Rheum Dis 2009;68:1673-1679

[25] Felson D, McLaughlin S,Goggins J, LaValley M, Gale E, Totterman S, Li W, Hill C, Gale D. Bone marrow edema and its relation to progression of knee osteoarthritis. Ann Intern Med. 2003;139:330-336

[26] Hill C. Knee effusions, popliteal cysts, and synovial thickening: association with knee pain in osteoarthritis. J Rheumatol 2001;28:1330-1337

[27] Valdes A, De Wilde G, Doherty S, Lories R, Vaughn F, Laslett L, Maciewicz r, Soni A, Hart D, Zhang W, Muir K, Dennison E, Wheeler M, Leaverton P, Cooper C, Spector T, Cicuttini F, Chapman V, Jones G, Arden N, Doherty M. The Ile585Val TRPV1 variant is involved in risk of painful knee osteoarthritis. Ann Rheum Dis 2011;70:1556-1561

[28] Richette P, Bardin T. Gout. Lancet 2010;375:318-328 
[29] Lohmander L, Ostenberg A, Englund M, Roos H. High Prevalence of Knee Osteoarthritis, Pain, and Functional Limitations in Female Soccer Players Twelve Years After Anterior Cruciate Ligament Injury. Arthritis Rheum 2004;50:3145-3152

[30] Mine T, Kimura M, Sakka A, Kawai S. Innervation of nociceptors in the menisci of the knee joint: an immunohistochemical study. Arch Orthop Trauma Surg 2000;120:201204

[31] Berthiaume M, Raynauld J, Martel-Pelletier J, Labonte F, Beaudoin G, Bloch D, Choquette D, Haraoui B, Altman R, Hochberg M, Meyer J, Cline G, Pelletier J. Meniscal tear and extrusion are strongly associated with progression of symptomatic knee osteoarthritis as assessed by quantitative magnetic resonance imaging. Ann Rheum Dis 2005;64:556-563

[32] Roos H, Lauren M, Adalberth T, Roos E, Jonsson K, Lohmander L. Knee osteoarthritis after meniscectomy: Prevalence of radiographic changes after twenty-one years compared with matched controls. Arthritis Rheum 1998;41:687-693

[33] Englund M, Lohmander L. Risk factors for symptomatic knee osteoarthritis fifteen to twenty two years after meniscectomy. Arthritis Rheum 2004;50:2811-2819

[34] Wiberg G. Roentgenographic and anatomical studies on the patellofemoral joint: With special reference to chondromalacia patella Acta Orthop Scand 1941;12:319

[35] Dye S. The pathophysiology of patellofemoral pain: a tissue homeostasis perspective. Clin Orthop Relat Res 2005;436:100-110

[36] Stanitski C. Anterior knee pain syndromes in the adolescent. J Bone Joint Surg Am 1993;75-A:1407-1416

[37] Kodali, P., A. Islam, and J. Andrish, Anterior knee pain in the young athlete, diagnosis and treatment. Sports Medicine, Arthroscopy Review, 2011. 19: p. 27-33.

[38] Saupe E. Primäre knochenmarkseiterung der kniescheibe. Deutsche Z Chir 1943;258:3869

[39] Nimon G, Murray D, Sandow M, Goodfellow J. Natural history of anterior knee pain: a 14- to 20-year follow-up of nonoperative management. J Pediatr Orthop. 1998;18:11822.

[40] Schiphof D, de Klerk B, Koes B, Bierma-Zeinstra S. Good reliability, questionable validity of 25 different classification criteria of knee osteoarthritis: a systematic appraisal. Journal of Clinical Epidemiology 2008;61:1205-1215

[41] Post W. Anterior knee pain: Diagnosis and treatment. J Am Acad Orthop Surg 2005;13:534-543

[42] Cutbill J, Ladly K, Bray R, Thorne, P, Verhoef M. Anterior knee pain: A review. Clin J Sports Med 1997;7:40-45

[43] Murray TF, Dupont JY Fulkerson JP. Axial and lateral radiographs in evaluating patellofemoral malalignment. Am J Sports Med 1999;27:580-4

[44] Insall J, Salvati E. Patella position in the normal knee joint. Radiology 1971; 101:1014 
[45] Egund N. The radiographic axial view of the patellofemoral joint: examination in the supine or standing position. Eur Radiol 2001;11:130

[46] Muhle C. Brossman J. Heller M. Kinematic CT and MR imaging of the patellofemoral joint. Eur Radiol 9 508-18 1999

[47] Brossman J, Muhle C, Bull CC, Zieplies J, Melchert UH, Brinkmann G, Schroder C, Heller M Cine MR imaging before and after realignment surgery for patellar maltracking comparison with axial radiographs. Skeletal radiology 24: 191-6 1995

[48] Sofat N, Hamann P, Barrick TR, Howe FA. Activation of central pain pathways in rheumatic diseases: What we have learned from functional neuroimaging studies. Current rheumatology reviews, 2010,6;3

[49] Baliki M, Katz J, Chialvo DR, Apkarian AV. Single subject pharmacological-MRI (fMRI) study: modulation of brain activity of psoriatic arthritis pain by cycloxygenase-2 inhibitor. Mol pain 2005;2(1):32

[50] Gwilym S, Keltner JR, warnaby CE, et al. Pschyosocial and functional imaging evidence supporting the presence of central sensitization in a cohort of osteoarthritis patients. arthritis rheum 2009; 61(9): 1226-1234

[51] Lee GW, Weeks PM: The role of bone scintigraphy in diagnosing reflex sympathetic dystrophy. J Hand Surg [Am] 1995;20:458-63.

[52] Van Holsbeeck M, Intracaso JH. Musculoskeletal ultrasonography. Radiol Clin North Am 1992; 30:907-925

[53] Ptasnik R, Feller J, Bartlett J, Fitt G, Mitchell A, Hennessy O. The value of sonography in the diagnosis of traumatic rupture of the anterior cruciate ligament of the knee. AJR 1995; 164:1461-1463.

[54] L. Friedman K. Finlay E. Jurriaans Skeletal Radiol (2001) 30:361-377

[55] Bianchi S, Abdelwahab IF, Zwass A et al. Diagnosis of tears of the quadriceps tendon of the knee: value of sonography. Am J Roentgenol 1994; 162:1137

[56] Jacobson JA, van Holsbeeck MT. Musculoskeletal ultrasonography. Orthop Clin N Amer 1998; 29:135-167)

[57] Mcalindon TE, Watt I, McCrae F, Goddard P, Dieppe PA. magnetic resonance imaging in osteoarthritis of the knee: correlation with radiographic and scintigraphic findings. Ann Rheum disease 1991;50:14-9

[58] Zanetti M, Bruder E, Romero J, Hodler J. Bone marrow edema pattern in osteoarthritic knees: Correlation between MR imaging and histological findings. Radiology 2000; 215:835-840

[59] Felson DT, Chaisson CE, Hill CL, Totterman SM, Gale ME, Skinner KM, et al. The association of bone marrow lesions with pain in knee osteoarthritis. Ann Intern med 2001;134:541-9

[60] Tanamas et al. Bone marrow lesions in people with knee osteoarthritis predeict progression of disease and joint replacement: a longitudinal study. Rheumatology 2010;49:2413-2419 
[61] Hefti F, Beguiristain J, Krauspe R, Möller-Madsen B, Riccio V, Tschauner C, Wetzel R, Zeller R.J Osteochondritis dissecans: a multicenter study of the European Pediatric Orthopedic Society. Pediatr Orthop B. 1999 Oct;8(4):231-45.

[62] De Smet AA, Ilahi OA, Graf BK. Untreated osteochondritis dissecans of the femoral condyles: prediction of patient outcome using radiographic and MR findings. Skeletal Radiol. 1997 Aug;26(8):463-7.

[63] Dupont JY. Synovial plicae of the knee. Controversies and review. Clin Sports Med. 1997 Jan;16(1):87-122.

[64] Medial plica syndrome. Sznajderman T, Smorgick Y, Lindner D, Beer Y, Agar G. Isr Med Assoc J. 2009 Jan;11(1):54-7.

[65] Jacobson JA, Lenchik L, Ruhoy MK, Schweitzer ME, Resnick D. MR imaging of the infrapatellar fat pad of Hoffa. Radiographics. 1997 May-Jun;17(3):675-91.

[66] Elias DA, White LM. Imaging of patellofemoral disorders. Clinical radiology 2004;59: 543-557

[67] Muckle DS, Minns RJ. Biological response to woven carbon fibre pads in the knee. A clinical and experimental study. J Bone Joint Surg Br. 1990 Jan;72(1):60-2.

[68] Kodali P, Islam A, Andrish J. Anterior Knee Pain in the Young Athlete Diagnosis and Treatment. Sports Med Arthrosc Rev 2011;19:27-33

[69] Nomura E. Classification of lesions of the medial patello-femoral ligament in patellar dislocation. Int Orthop. 1999;23(5):260-3.

[70] Quinn SF, Brown TR, Demlow TA. MR imaging of patellar retinacular ligament injuries. J Magn Reson Imaging. 1993 Nov-Dec;3(6):843-7.

[71] Yusuf E, Kortekaas MC, Watt I, Huizinga TW, Kloppenburg M. Do knee abnormalities visualised on MRI explain knee pain in knee osteoarthritis? A systematic review. Ann Rheum Dis. 2011 Jan;70(1):60-7.

[72] Bolgla, L.A. and M.C. Boling, Systematic review of the literature: an update for the conservative management of patellofemoral pain syndrome: a systematic review of the literature from 2000 to 2010. The International Journal of Sports Physical Therapy, 2011. 6(2): p. 112-125.

[73] Witvrouw E, Lysens R, Bellemans J, Peers K, Vanderstraeten G. Open versus closed kinetic chain exercises for patellofemoral pain. A prospective, randomized study. Am J Sports Med. 2000 Sep-Oct;28(5):687-94.)

[74] Wilk KE, Escamilla RF, Fleisig GS, Barrentine SW, Andrews JR, Boyd ML. A comparison of tibiofemoral joint forces and electromyographic activity during open and closed kinetic chain exercises. Am J Sports Med. 1996 Jul-Aug;24(4):518-27.

[75] Escamilla RF, Fleisig GS, Zheng N, Barrentine SW, Wilk KE, Andrews JR. Biomechanics of the knee during closed kinetic chain and open kinetic chain exercises. Med Sci Sports Exerc. 1998 Apr;30(4):556-69.

[76] Tang SF, Chen CK, Hsu R, Chou SW, Hong WH, Lew HL. Vastus medialis obliqus and vastus lateralis activity in open and closed kinetic chain exercises in patients with 
patellofemoral pain syndrome: an electromyographic study. Arch Phys Med Rehabil. 2001 Oct;82(10):1441-5.

[77] Souza RB, Draper CE, Fredericson M, Powers CM. Femur rotation and patellofemoral joint kinematics: a weight-bearing magnetic resonance imaging analysis. J Orthop Sports Phys Ther. 2010 May;40(5):277-85.

[78] Tiberio D. Evaluation of functional ankle dorsiflexion using subtalar neutral position. A clinical report. JOSPT Phys Ther. 1987 Jun;67(6):955-7

[79] Collins N, Crossley KM, Beller E, Darnell R, McPoil T, Vicenzino B. Foot orthoses and physiotherapy in the treatment of patellofemoral pain syndrome: randomized clinical trial.Br J Sports med 2009;43(3):169-171

[80] Crossley KM, Bennell KL, Green S, Cowan SM, McConnel J. Physical therapy for patellofemoral pain. A randomized double blinded, placebo-controlled trial. Am J sports med 2002;30(6):857-865

[81] Lun VM, Wiley JP, Meeuwisse WH, Yanagawa TL. Effectiveness of patellar bracing for treatment of patellofemoral pain syndrome. Clin J sport med 2005;35(4):235-240

[82] Whitelaw GP Jr, Rullo DJ, Markowitz HD, Marandola MS, DeWaele MJ. A conservative approach to anterior knee pain. Clin Orthop Relat Res. 1989 Sep;(246):234-7

[83] Kannus P, Natri A, Paakkala T, Järvinen M. An outcome study of chronic patellofemoral pain syndrome. Seven-year follow-up of patients in a randomized, controlled trial. J Bone Joint Surg Am. 1999 Mar;81(3):355-63.

[84] Doherty $M$ et al. A randomised controlled trial of ibuprofen, paracetamol or a combination tablet of ibuprofen/paracetamol in community-derived people with knee pain. Ann Rheum Dis 2011;70:1534-1541.

[85] Berenbaum F. Osteoarthritis year 2010 in review: Pharmacological therapies. Osteorthritis and cartilage 19 (2011) 361-365.

[86] Bannuru RR, Natov NS, Dasi UR, Schmid CH, McAlindon TE. Therapeutic trajectory following intra-articular hyaluronic acid injection in knee osteoarthritis--meta-analysis. Osteoarthritis Cartilage. 2011 Jun;19(6):611-9.

[87] Schnitzer TJ, Lane NE, Birbara C, Smith MD, Simpson SL, Brown MT. Long-term openlabel study of tanezumab for moderate to severe osteoarthritic knee pain. Osteoarthritis Cartilage. 2011 Jun;19(6):639-46.

[88] Bliddal et al. Weight loss as treatment for knee osteoarthritis symptoms in obese patients: 1-year results from a randomised controlled trial. Ann Rheum Dis 2011;70:1798-1803.

[89] Dieppe P, Lim K, Lohmander S. Who should have knee joint replacement surgery for osteoarthritis?. Int J Rheum Dis. 2011 May;14(2):175-80.

[90] T. Dixona, M.E. Shawb, P.A. Dieppe. Analysis of regional variation in hip and knee joint replacement rates in England using Hospital Episodes Statistics. Public Health. 2006;120(1) 83-90 
[91] Riddle DL, Keefe FJ, Nay WT, McKee D, Attarian DE, Jensen MP. Pain coping skills training for patients with elevated pain catastrophising who are scheduled for knee arthroplasty: a quasi-experimental study. Arch phys rehabil. 2011 Jun;92(6):859-65.) 